\title{
Modification of the Alere GIARDIA Ag TEST immunochromatography KIT methodology for its use in frozen fecal sediment of dogs and cats
}

\author{
VIVIANE A.N. COSTA ${ }^{1}$, BEATRIZ BRENER ${ }^{2}$, ANA BEATRIZ M. FONSECA ${ }^{3}$ and ADRIANA P. SUDRÉ ${ }^{2}$ \\ ${ }^{1}$ Programa de Pós-Graduação em Microbiologia e Parasitologia Aplicadas, Instituto Biomédico, Universidade \\ Federal Fluminense, Rua Professor Ernani Melo, 101, São Domingos, 24210-130 Niterói, RJ, Brazil \\ ${ }^{2}$ Departamento de Microbiologia e Parasitologia, Instituto Biomédico, Universidade Federal \\ Fluminense, Rua Professor Ernani Melo, 101, São Domingos, 24210-130 Niterói, RJ, Brazil \\ ${ }^{3}$ Departamento de Estatística, Instituto de Matemática e Estatística, Universidade Federal \\ Fluminense, Rua Mário Santos Braga, s/n, Valonguinho, 24020-140 Niterói, RJ, Brazil
}

Manuscript received on October 10, 2016; accepted for publication on January 11, 2017

\begin{abstract}
Giardia duodenalis is a worldwide intestinal parasite and is one of the most frequent protozoa species infecting dogs and cats. This study aimed to modify the methodology of Alere GIARDIA Ag TEST KIT for its use in frozen fecal sediments with different storage times in a freezer $\left(-20^{\circ} \mathrm{C}\right)$, thus expanding the range of use of this methodology. One hundred fecal sediments from dogs $(n=50)$ and cats $(n=50)$ previously examined by optical microscopy for Giardia cysts were selected for this study. The agreement between the modified immunochromatography and microscopy results was calculated by Kappa coefficient. To evaluate the performance of the modified immunochromatography assay on samples with different storage time, the fecal sediments were divided into three groups according to the time of storage in a freezer: (a) $\leq 1$ year $(n=37)$; $(b)>1$ year and $\leq 3$ years $(n=39)$; $(c)>10$ years (max. 13 years) $(n=24)$. The results obtained by the modified immunochromatography assay demonstrates a higher sensitivity of this technique when compared with microscopy, regardless of the frozen storage time. These results allow for the use of this methodology in a greater scope of analysis, especially in frozen fecal sediment triage in sample collections, enabling epidemiological and comparative analysis along different decades.
\end{abstract}

Keywords: cats, diagnosis, dogs, Giardia duodenalis, immunochromatography.

\section{INTRODUCTION}

Giardia duodenalis is a worldwide intestinal protozoa that can infect a wide variety of mammal hosts, including humans, and is one of the most frequent protozoa species infecting dogs and cats (Thompson et al. 2000, Ballweber et al. 2010).

Correspondence to: Adriana Pittella Sudré

E-mail: asudre@id.uff.br
Giardia infection rates are variable among those hosts, and are associated with a number of factors, such as, study design, geographical location, climate and season, diagnostic methodology, studied population, health status of the host and animal management conditions (Dixon et al. 1997).

Microscopy is the gold standard methodology for Giardia duodenalis diagnosis (Payne and Artzer 2009). However, this method has a high 
percentage of false-negative results because of the intermittence, low number and size of the cysts shed in fecal samples. Thus, a combination of diagnostic methods is frequently indicated to overcome such limitations (Tangtrongsup and Scorza 2010). Several complementary diagnostic methods have emerged in the past years, especially immunological methods, as tools to improve Giardia diagnosis.

The immunochromatography kit ALERE GIARDIA Ag TEST KIT ${ }^{\circledR}$ is a method developed for rapid qualitative diagnosis of Giardia duodenalis cyst antigens in fecal samples from dogs and cats by using a swab for sample collection. However, Current and Garcia (1991) found that parasites have a heterogeneous distribution in a fecal sample, so the use of a swab for collection of a fragment of solid or semi-solid feces could result in false-negative tests, especially in low parasite burdens. Therefore, the present study aimed to modify the immunochromatography test to allow its use in fecal sediments of dogs and cats. Moreover, the performance of this modified technique was evaluated on samples from different storage freezing time in order to expand the use of this test to samples (fecal sediment) belonging to research laboratory collections, allowing epidemiological analysis and comparisons between different years.

\section{MATERIALS AND METHODS}

One hundred frozen fecal sediments from dogs $(\mathrm{n}=50)$ and cats $(\mathrm{n}=50)$ belonging to two collection of samples from research laboratories of the Universidade Federal Fluminense were selected. Fecal sediments were previously obtained using concentration techniques usually employed in parasitological exams (centrifuge-sedimentation or spontaneous sedimentation). All samples were previously examined by optical microscopy for Giardia duodenalis cysts. Among those, 18 samples
(6 from dogs and 12 from cats) were positive for Giardia duodenalis cysts by microscopy exam.

To evaluate the performance of the modified immunochromatography on samples with different storage time in a freezer $\left(-20^{\circ} \mathrm{C}\right)$, fecal sediments were divided in three groups according to the time of storage by freezing: (a) $\leq 1$ year $(n=37)$; (b) $>$ 1 year and $\leq 3$ years $(n=39)$; (c) $>10$ years $(\max$. 13 years) $(n=24)$. The freezing times of the chosen samples were based on the availability of samples in the laboratory collection.

Because the immunochromatographic test was developed for use in fresh feces by collecting a fecal fragment with a swab, the modification to allow its use in fecal sediments consisted in not using the swab for sample collection, but homogenizing $50 \mu \mathrm{l}$ of fecal sediment with $50 \mu 1$ of the kit dilution buffer for approximately 10 seconds. The following steps were performed according to the manufacturers' recommendations, which consisted of removing a sample of the supernatant using the pipette provided by the kit manufacturer followed by the instillation of 4 drops of the sample in the kit cassette well. Two readings of test results were recorded 5 and 10 minutes after the procedure. Each sample was tested in duplicate.

The agreement between the modified immunochromatography and microscopy results was calculated by Kappa coefficient (Smith 1995). The evaluation of the influence of storage time on the performance of the modified method was done by Mann-Whitney test at a significance level of 5\%.

\section{RESULTS}

The immunochromatography test was positive for Giardia duodenalis antigens in 29 samples (29\%), 12 from dogs $(24 \% ; 12 / 50)$ and 17 from cats $(34 \%$; 17/50). A comparison between microscopy and immunochromatography results is shown on table I.

The frequency of agreement was $74 \%$ between microscopy and immunochromatography. The 


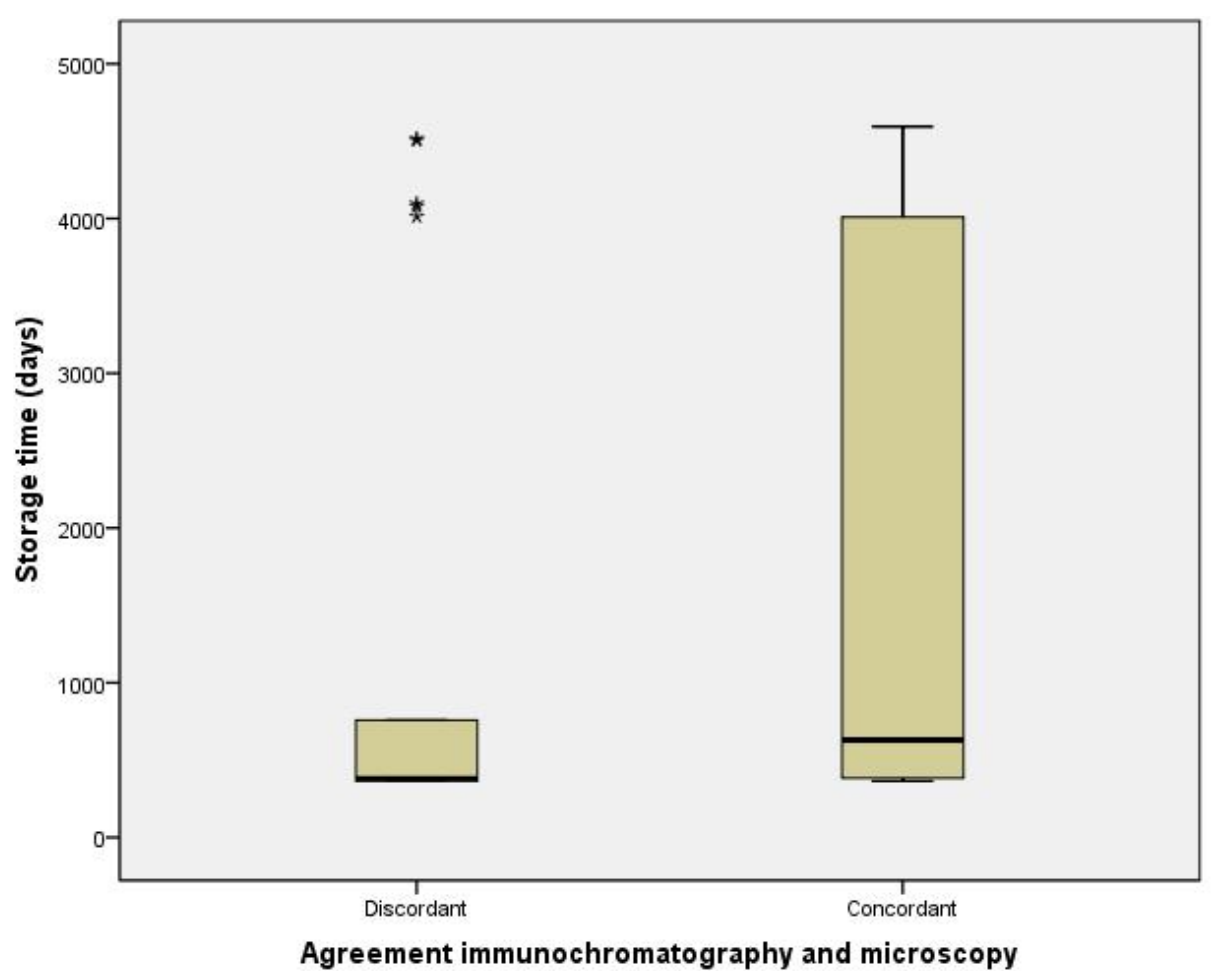

Figure 1 - Distribution of concordant results between microscopy and immunochromatography according to frozen storage time.

TABLE I

Comparison of relative $(\%)$ and absolute frequencies of microscopy and modified immunochromatography results for Giardia duodenalis diagnosis.

\begin{tabular}{lllcc}
\hline \multirow{2}{*}{ Positive } & & \multicolumn{2}{c}{ Immunochromatography } & \multirow{2}{*}{ Total } \\
\cline { 3 - 4 } & & Negative & \\
\hline \multirow{2}{*}{ Microscopy } & Positive & $11 \%(11)$ & $7 \%(7)$ & $18 \%(18)$ \\
& Negative & $18 \%(18)$ & $64 \%(64)$ & $82 \%(82)$ \\
& & $29 \%(29)$ & $71 \%(71)$ & $100 \%(100)$ \\
\hline
\end{tabular}

kappa coefficient showed a fair agreement between both methods $(\kappa=0.32)$.

Figure 1 demonstrates the distribution of concordant results between both tests (immunochromatography and microscopy) in relation to the storage time. The discordant results were mostly observed on samples with a lesser freezing time. In these, 3 (23\%) were positive on microscopy and negative on immunochromatography and 10 (77\%) were negative on microscopy and positive on immunocrhomatography.
The results obtained by the modified immunochromatography demonstrate a higher sensitivity of this technique when compared with microscopy, regardless of the frozen storage time. Moreover, 18/29 samples were positive only by immunochromatography, contributing to a low level of concordance between the techniques evaluated.

\section{DISCUSSION}

A higher sensitivity of immunological methods when compared to conventional microscopy have 
been demonstrated by several studies in a variety of hosts (Vidal and Catapani 2005, Garcia and Garcia 2006, Geurden et al. 2008, Ignatius et al. 2014). Such superiority could be explained by several factors, mainly by the capacity of detection of minimal quantities of antigens, even when the parasite burden is low, which would require several fecal samples for its detection by conventional microscopy methods, especially on chronic infections where the cyst shedding intermittency is frequently observed (Mohammed Mahdy et al. 2008). Moreover, the freezing and thawing of fecal samples may result in damage to cysts, thus, impairing the usefulness of microscopy with frozen samples.

It is worth noting that the usage of fecal sediment rather than the fecal sample swab could have favoured the diagnosis of samples with low cyst quantities, because of the concentration necessary for obtaining the fecal sediment. Moreover, the coproantigen detection can be performed even if the parasite is not intact in the sample, which could not be done by traditional microscopy.

However, it was not possible to detect antigens in seven samples that had cysts of Giardia sp. by microscopy exam, which also contributed to the low concordance of methods. Garcia and Garcia (2006) and Weitzel et al. (2006) also reported these discordant results. One possible explanation is the genetic heterogeneity of Giardia sp. isolates from different hosts (Mekaru et al. 2007). Additionally, possible reading errors during microscopy exam cannot be discarded because of the human error inherent to these techniques. Dixon et al. (1997) and Vidal and Catapani (2005) stated that the experience and the fatigue level of microscopists and the presence of a great amount of debris on the slides could influence the results of a microscopy analysis.

Although the immunochromatographic test allows a faster diagnosis (about 20 minutes) when compared with microscopy (about 1 to 2 hours, depending on the technique used) and does not require special equipment for diagnosis, it still has higher cost (about 5 times more).

The discordance between microscopy and immunochromatography results were mostly observed on samples with a lesser freezing time. However, $77 \%$ of these discordant samples were negative in microscopy and positive in immunochromatography. Such a result can be explained by a greater sensitivity of the immunochromatographic method compared to microscopy in samples with a low number of cysts, and thus there is no correlation with the freezing time.

Therefore, the modification of the ALERE GIARDIA Ag immunochromatography TEST KIT $^{\circledR}$ showed good performance regardless of frozen storage time. These results allow for the use of this methodology in a greater scope of analysis, especially in frozen fecal sediment triage in sample collections, enabling epidemiological and comparative analysis along different decades.

\section{ACKNOWLEDGMENTS}

The authors would like to thank Rita Cúbel and Tatiana Xavier from Universidade Federal Fluminense for providing a subset of the fecal sediments used in the study. To the ALERE/ TECNEW company for the donation of the immunochromatographic kits used in the present study. To Universidade Federal Fluminense (UFF), Coordenação de Aperfeiçoamento de Pessoal de Nível Superior (CAPES) and Fundação Carlos Chagas Filho de Amparo à Pesquisa do Estado do Rio de Janeiro (FAPERJ) for financial support.

\section{REFERENCES}

BALLWEBER LR, XIAO L, BOWMAN DD, KHAN G AND CAMA VA. 2010. Giardiasis in dogs and cats: update on epidemiology and public health significance. Trends Parasitol 26(4): 180-189. 
CURRENT WL AND GARCIA LS. 1991. Cryptosporidiosis. Clin Laboratory Med 11: 873-897.

DIXON BR, PARENTEAU M, MARTINEAU C AND FOURNIER J. 1997. A comparison of conventional micrsocopy, immunofluorrescence microscopy and flow cytometry in the detection of Giardia lamblia cysts in beaver fecal samples. J Immunol Methods 202: 27-33.

GARCIA LS AND GARCIA JP. 2006. Detection of Giardia lamblia Antigens in Human Fecal Specimens by a SolidPhase Qualitative Immunochromatographic Assay. J Clin Microbiol 44(12): 4587-4588.

GEURDENT,BERKVENSD, CASAERTS, VERCRUYSSE J AND CLAEREBOUT E. 2008. A Bayesian evaluation of three diagnostic assays for the detection of Giardia duodenalis in symptomatic and asymptomatic dogs. Vet Parasitol 157(1-2): 14-20.

IGNATIUS R, GAHUTU JB, KLOTZ C, MUSEMAKWERI A, AEBISCHER T AND MOCKENHAUPT FP. 2014. Detection of Giardia duodenalis assemblage A and B isolates by immunochromatography in stool samples from Rwandan children. Clin Microbiol Infect 20: 783-784.

MEKARU SR, MARKS SL, FELLEY AJ, CHOUICHA $\mathrm{N}$ AND KASS PH. 2007. Comparison of direct immunofluorescence, immunoassay, and fecal flotation for detection of Cryptosporidium spp. and Giardia spp. in naturally exposed cats in four Northern California animal shelters. J Vet Inter Med 21: 959-965.
MOHAMMED MAHDY AK, LIM YAL, SURIN J, WAN KL AND AL-MEKHLAFI MSH. 2008. Risk factors for endemic giardiasis: highlighting the possible association of contaminated water and food. Trans R Soc Trop Med Hygiene 102: 465-470.

PAYNE P AND ARTZER M. 2009. The biology and control of Giardia spp. and Tritrichomonas foetus. Vet Clin N Am Small 39(6): 993-1007.

SMITH RD. 1995. Veterinary Clinical Epidemiology: A problem-Oriented Approach, $2^{\text {nd }}$ ed., CCR Press, $279 \mathrm{p}$.

TANGTRONGSUP S AND SCORZA V. 2010. Update on the diagnosis and management of giardia spp. infections in dogs and cats. Topics in Companion Animal Medicine 25(3): 155-162.

THOMPSON RCA, HOPKINS RA AND HOMAN WL. 2000. Nomenclature and genetic groupings of Giardia infecting mammals. Parasitol 16: 210-218.

VIDAL AMB AND CATAPANI WR. 2005. Enzyme-linked immunosorbent assay (ELISA) immunoassaying versus microscopy: advantages and drawbacks for diagnosing giardiasis. São Paulo Med J 123(6): 282-285.

WEITZEL T, DITTRICH S, MÖHL I, ADUSU E AND JELINEK T. 2006. Evaluation of seven commercial antigen detection tests for Giardia and Cryptosporidium in stool samples. Clin Microbiol Infect 12(7): 656-659. 